\title{
ELECTRON INERTIAL EFFECTS ON RAPID ENERGY REDISTRIBUTION AT MAGNETIC X-POINTS
}

\section{Short Title: Electron inertial effects at X-points}

\author{
K. G. McCLEMENTS \\ UKAEA Culham Division, Culham Science Centre, Abingdon, \\ Oxfordshire, OX14 3DB, UK; k.g.mcclements@ukaea.org.uk
}

\author{
A. THYAGARAJA \\ UKAEA Culham Division, Culham Science Centre, Abingdon, \\ Oxfordshire, OX14 3DB, UK; a.thyagaraja@ukaea.org.uk \\ N. BEN AYED \\ University of Bristol, H.H.Wills Physics Laboratory, Royal Fort, \\ Tyndall Avenue, Bristol, BS8 1TL, UK; nb1392@bris.ac.uk

\section{AND} \\ L. FLETCHER
}

University of Glasgow, Department of Physics and Astronomy, Glasgow, G12 8QQ, UK; lyndsay@astro.gla.ac.uk 


\begin{abstract}
The evolution of non-potential perturbations to a current-free magnetic Xpoint configuration is studied, taking into account electron inertial effects as well as resistivity. Electron inertia is shown to have a negligible effect on the evolution of the system whenever the collisionless skin depth is less than the resistive scale length. Non-potential magnetic field energy in this resistive MHD limit initially reaches equipartition with flow energy, in accordance with ideal MHD, and is then dissipated extremely rapidly, on an Alfvénic timescale that is essentially independent of Lundquist number. In agreement with resistive MHD results obtained by previous authors, the magnetic field energy and kinetic energy are then observed to decay on a longer timescale and exhibit oscillatory behavior, reflecting the existence of discrete normal modes with finite real frequency. When the collisionless skin depth exceeds the resistive scale length, the system again evolves initially according to ideal MHD. At the end of this ideal phase, the field energy decays typically on an Alfvénic timescale, while the kinetic energy (which is equally partitioned between ions and electrons in this case) is dissipated on the electron collision timescale. The oscillatory decay in the energy observed in the resistive case is absent, but short wavelength structures appear in the field and velocity profiles, suggesting the possibility of particle acceleration in oppositely-directed current channels. The model provides a possible framework for interpreting observations of energy release and particle acceleration on timescales down to less than a second in the impulsive phase of solar flares.
\end{abstract}

\title{
1 Introduction
}

Renewed interest in the long-standing problem of energy release in solar flares has been kindled by the launch of the Ramaty High Energy Solar Spectroscopic Imager (RHESSI) spacecraft (Lin et al. 2002) and, very recently, by observations of exceptionally large flares (Bowler 2003). Resistive magnetohydrodynamics (MHD) provides the framework for most theoretical studies of this problem (for a recent review, see Priest \& Forbes 2000). Craig \& McClymont (1991, 1993) and Craig \& Watson (1992), for example, have invoked the resistive MHD relaxation of a twodimensional magnetic X-point as a paradigm for energy release in flares. The magnetic equilibrium configuration in this analysis was potential (and thus stable), and the system was assumed to have a circular boundary centered on the X-point. Craig \& McClymont $(1991,1993)$ investigated the stable mode spectrum of such a configuration. A countably infinite set of discrete normal modes was identified, with azimuthally symmetric modes corresponding to topological reconnection. Both the real frequency and the damping rate were found to increase with the number of radial nodes. It was inferred that the spectrum after a sufficiently long time would be dominated by the mode with the longest radial wavelength, i.e. the lowest damping rate, regardless 
of the initial configuration. This damping rate was shown analytically to be given approximately by

$$
\gamma_{\text {disc }} \simeq \frac{\pi^{2} c_{A 0}}{2 R_{0}(\ln S)^{2}},
$$

where $S$ is the Lundquist number at the radial boundary of the system $R=R_{0}$ and $c_{A 0}$ is the Alfvén speed at that boundary, computed using the equilibrium magnetic field. The logarithmic dependence of the dissipation rate on $S$ is reminiscent of that predicted by the Petschek "fast" reconnection model (Petschek 1964), although it should be noted that Petschek used a steady state approach while the treatment of Craig \& McClymont was time-dependent. The latter authors noted that, for typical solar parameters, equation (1) yields an energy release timescale of the order of several minutes to an hour, This is sufficiently rapid to explain thermal energy release in the gradual phase of a flare, but too slow to account for the impulsive phase: as noted by Tandberg-Hanssen and Emslie (1989), the thick target interpretation of flare hard $\mathrm{X}$-ray bursts requires a field of the order of $0.02 \mathrm{~T}$ to be completely annihilated in a volume of around $10^{21} \mathrm{~m}^{3}$ every second.

Recognizing that resistive MHD may not provide a full description, several authors have investigated the possible role of non-MHD effects in flare energy release. Craig \& Watson (2003), for example, have identified exact reconnection solutions that include Hall current and electron inertial effects. Such effects have been studied extensively by researchers in the magnetospheric community: Birn et al. (2001) have summarised the results of a recent project to compare computational models of reconnection in Harris-type geometry ranging from resistive MHD to particle-in-cell. In a recent paper McClements \& Thyagaraja (2004) generalized the spectral analysis of Craig \& McClymont $(1991,1993)$ to include electron inertia. A key motivation for doing so in the laboratory context is that reconnection events in magnetic fusion experiments, such as those responsible for "sawtooth" oscillations in tokamaks, cannot be explained using MHD alone. As noted by Wesson (1991), the observed relaxation time in a sawtooth crash can be much shorter than the timescale implied by purely resistive models. McClements \& Thyagaraja used the following form of Ohm's law:

$$
\mathbf{E}+\mathbf{v} \times \mathbf{B}=\eta \mathbf{j}+\frac{m_{e}}{n e^{2}} \frac{\partial \mathbf{j}}{\partial t},
$$

where $\eta$ is resistivity, $n$ is particle density, and $m_{e}$, $e$ denote the electron mass and charge. McClements \& Thyagaraja demonstrated that the magnetic X-point eigenmode spectrum in this case has a continuous component in addition to the discrete spectrum studied by Craig \& McClymont. This continuum replaces the ideal MHD Alfvén continuum which is present when both resistivity and electron inertia are excluded. All of the finite frequency continuum modes have the same intrinsic damping rate, namely

$$
\gamma_{\mathrm{cont}}=\frac{c_{A 0}}{2 R_{0} S \delta_{e}^{2}}
$$


where $\delta_{e} \equiv c /\left(\omega_{p e} R_{0}\right), c$ being the speed of light and $\omega_{p e}$ the electron plasma frequency, is the collisionless skin depth normalized to the system size. Because $\delta_{e}$ is typically very small, the continuum mode damping rate given by equation (3) can be high in absolute terms. In fact, if the resistivity is determined by electron-ion collisions, $\gamma_{\text {cont }}$ is essentially equal to the electron collision frequency

$$
\nu_{e} \simeq 30 \frac{n_{15}}{T_{6}^{3 / 2}} \mathrm{~s}^{-1}
$$

where $n_{15}$ is particle density in units of $10^{15} \mathrm{~m}^{-3}$ and $T_{6}$ is electron temperature in units of $10^{6} \mathrm{~K}$. Thus, for typical solar coronal parameters and Spitzer resistivity, the energy dissipation timescale implied by equation (3) is a fraction of a second. Statistically significant fluctuations have been observed in flare hard X-ray emission on such timescales (e.g. Kiplinger et al. 1983): this suggests that non-MHD effects may be significant in the context of flare energy release. However, in order to compute dissipation timescales reliably, it is appropriate to solve an initial value problem rather than using an eigenvalue approach. Craig \& Watson (1992) performed such an analysis using a resistive MHD model: in this paper we adopt a similar approach, but with the electron inertial term in Ohm's law taken into account. Specifically, we solve a set of linearized equations describing a perturbed magnetic X-point using parameters that range from the resistive MHD limit considered by Craig \& Watson (1992) to the collisionless limit. Our approach differs from the two-fluid analyses carried out for example by Biskamp, Schwarz, \& Drake (1997) and Ramos, Porcelli \& Verástegui (2002) in that we focus on a single, dominant non-MHD effect, namely electron inertia. Compared to resistive MHD, the problem is then characterised by only one additional parameter: the collisionless skin depth. Using this model, we address the key issue of how rapidly the energy in a non-potential magnetic field perturbation is dissipated or converted to kinetic energy, making an exact comparison with the resistive MHD scenario investigated by Craig \& Watson (1992).

This paper is structured as follows. After formulating the linearized initial value problem in $\S 2$, we proceed to solve it analytically and numerically for various parameter regimes in $\S 3$. The possible relevance of these solutions to energy release in solar flares is discussed in $\S 4$.

\section{Formulation of Initial Value Problem}

We consider inviscid incompressible perturbations of a two-dimensional current-free magnetic X-point in the low plasma beta limit. With Ohm's law given by equation

(2), the induction and momentum equations can be written in the form (McClements \& Thyagaraja 2004)

$$
\frac{\partial}{\partial t}\left(\psi-\frac{c^{2}}{\omega_{p e}^{2}} \nabla^{2} \psi\right)+(\mathbf{v} \cdot \nabla) \psi=\frac{\eta}{\mu_{0}} \nabla^{2} \psi
$$




$$
\frac{\partial \mathbf{v}}{\partial t}+(\mathbf{v} \cdot \nabla) \mathbf{v}=-\frac{1}{\mu_{0} \rho}\left(\nabla^{2} \psi\right) \nabla \psi
$$

where $\psi$ is a magnetic flux function whose curl yields the magnetic field, $\rho$ and $\mathbf{v}$ denote fluid density and velocity, and $\mu_{0}$ is vacuum permeability. Both the magnetic field and $\mathbf{v}$ are assumed to lie in the $(x, y)$ plane of the $\mathrm{X}$-point. We linearize these equations by neglecting terms of second order in $\mathbf{v}$ and putting $\psi=\psi_{E}+\tilde{\psi}$ where

$$
\psi_{E}=\frac{B_{0}}{2 R_{0}}\left(y^{2}-x^{2}\right)=-\frac{B_{0} R^{2}}{2 R_{0}} \cos 2 \theta,
$$

$\tilde{\psi}$ is assumed to be azimuthally symmetric, and $|\nabla \tilde{\psi}|$ is assumed to be much smaller than $\left|\nabla \psi_{E}\right|$. In equation (7) $B_{0}$ is the unperturbed magnetic field in the $(x, y)$ plane at radius $R=R_{0}$ and $\theta$ denotes azimuthal angle. When linearized, equations (5) and (6) become

$$
\begin{gathered}
\frac{\partial}{\partial t}\left(\tilde{\psi}-\frac{c^{2}}{\omega_{p e}^{2}} \nabla^{2} \tilde{\psi}\right)+(\mathbf{v} \cdot \nabla) \psi_{E}=\frac{\eta}{\mu_{0}} \nabla^{2} \tilde{\psi} \\
\frac{\partial \mathbf{v}}{\partial t}=-\frac{1}{\mu_{0} \rho_{0}}\left(\nabla^{2} \tilde{\psi}\right) \nabla \psi_{E},
\end{gathered}
$$

where $\rho_{0}$ denotes the unperturbed density (assumed to be uniform). These equations can be combined to give a single equation for $\tilde{\psi}$ in which $\theta$ does not appear except in the Laplacian operator (Craig \& McClymont 1991; McClements \& Thyagaraja 2004). In the linear approximation, the variation of $\tilde{\psi}$ with $\theta$ can thus be separated from the variation with $R$ and $t$. In this paper we concentrate on the azimuthally symmetric case in which $\tilde{\psi}$ is independent of $\theta$.

It is clear from equations (7) and (9) that the components of $\mathbf{v}$ are $\theta$-dependent even when $\tilde{\psi}$ is azimuthally symmetric. Formally integrating equation (9) with respect to time and writing the result explicitly in terms of $(R, \theta, z)$ components, we obtain

$$
\mathbf{v}=\frac{B_{0} R}{\mu_{0} \rho_{0} R_{0}}(\cos 2 \theta,-\sin 2 \theta, 0) \int \nabla^{2} \tilde{\psi} d t \equiv v(\cos 2 \theta,-\sin 2 \theta, 0)
$$

The function $v(R, t)$, defined in this way, satisfies the scalar momentum equation

$$
\frac{\partial v}{\partial t}=\frac{B_{0} R}{\mu_{0} \rho_{0} R_{0}} \nabla^{2} \tilde{\psi}
$$

and the radial and azimuthal velocity components are given by

$$
\begin{gathered}
v_{R}=v \cos 2 \theta, \\
v_{\theta}=-v \sin 2 \theta .
\end{gathered}
$$

It should be noted that $v$ in these expressions can be either positive or negative. The induction equation then becomes

$$
\frac{\partial}{\partial t}\left(\tilde{\psi}-\frac{c^{2}}{\omega_{p e}^{2}} \nabla^{2} \tilde{\psi}\right)-\frac{v B_{0} R}{R_{0}}=\frac{\eta}{\mu_{0}} \nabla^{2} \tilde{\psi}
$$


Following Craig \& McClymont (1991), we make the system dimensionless by normalising $R$ to $R_{0}, v$ to $c_{A 0} \equiv B_{0} /\left(\mu_{0} \rho_{0}\right)^{1 / 2}, t$ to $R_{0} / c_{A 0}$ and $\tilde{\psi}$ to $B_{0} R_{0}$. Dropping the tilde on $\tilde{\psi}$, we thus obtain

$$
\begin{gathered}
\frac{\partial}{\partial t}\left[\psi-\frac{\delta_{e}^{2}}{r} \frac{\partial}{\partial r}\left(r \frac{\partial \psi}{\partial r}\right)\right]=v r+\frac{1}{S r} \frac{\partial}{\partial r}\left(r \frac{\partial \psi}{\partial r}\right), \\
\frac{\partial v}{\partial t}=\frac{\partial}{\partial r}\left(r \frac{\partial \psi}{\partial r}\right),
\end{gathered}
$$

where $S \equiv \mu_{0} R_{0} c_{A 0} / \eta$. We proceed to solve these equations subject to initial conditions on $\partial \psi / \partial r$ and $v$, and boundary conditions on $\partial \psi / \partial r$. Regularity requires that the perturbation to the azimuthal magnetic field $-\partial \psi / \partial r$ vanish at $r=0$; this is consistent with the linear approximation since the equilibrium field in the $(x, y)$ plane has a null at the X-point. We also set $\partial \psi / \partial r=0$ at $r=1$, for reasons that will be explained in the next section. Following Craig \& Watson (1992), we consider both spatially extended initial field perturbations and localized ones. A simple obvious choice of initial condition for $v$, adopted throughout this paper, is $v=0$.

\section{Solution of Initial Value Problem}

\subsection{Energy Evolution}

Our principal objective is to compute the evolution of field and kinetic energy associated with a perturbed X-point. In the general case, with finite collisionless skin depth $\delta_{e}$, the total energy $\mathcal{E}$ has three components: non-potential field energy $\mathcal{E}_{\mathrm{f}}$, ion kinetic energy $\mathcal{E}_{\text {ki }}$ and electron kinetic energy $\mathcal{E}_{\mathrm{ke}}$. In terms of the dimensionless variables introduced in the previous section, these energy components can be represented by the integrals

$$
\begin{gathered}
\mathcal{E}_{\mathrm{f}}=\frac{1}{2} \int_{0}^{1}\left(\frac{\partial \psi}{\partial r}\right)^{2} r d r \\
\mathcal{E}_{\mathrm{ki}}=\frac{1}{2} \int_{0}^{1} v^{2} r d r \\
\mathcal{E}_{\mathrm{ke}}=\frac{1}{2} \int_{0}^{1} \delta_{e}^{2}\left(\nabla^{2} \psi\right)^{2} r d r .
\end{gathered}
$$

The identification of the integral in equation (17) as the non-potential field energy follows from the fact that the magnetic field perturbation is $-\partial \psi / \partial r$. Locally, there is a contribution to the field energy density proportional to $\left(\partial \psi_{E} / \partial r\right)(\partial \psi / \partial r)$, but this gives a zero contribution to the total energy when integrated over $\theta$ since $\partial \psi_{E} / \partial r$ is proportional to $\cos 2 \theta$ whereas $\partial \psi / \partial r$ is azimuthally symmetric. Ions make the dominant contribution to the bulk fluid velocity $\mathbf{v}$, and therefore it is appropriate to 
regard the integral in equation (18) as a measure of ion kinetic energy. On the other hand, most of the current is carried by electrons, and the current density (which is oriented in the $z$-direction) is proportional to $-\nabla^{2} \psi$ : it follows from this that the integral in equation (19) represents electron kinetic energy.

In the limit $S \rightarrow \infty$ the total energy

$$
\mathcal{E}=\mathcal{E}_{\mathrm{f}}+\mathcal{E}_{\mathrm{ki}}+\mathcal{E}_{\mathrm{ke}}=\frac{1}{2} \int_{0}^{1}\left[\left(\frac{\partial \psi}{\partial r}\right)^{2}+v^{2}+\delta_{e}^{2}\left(\nabla^{2} \psi\right)^{2}\right] r d r
$$

is conserved. To establish this result, we evaluate the time derivative of $\mathcal{E}$, using equations (15) and (16) to eliminate $\partial \psi / \partial t$ and $\partial v / \partial t$, obtaining

$$
\frac{d \mathcal{E}}{d t}=-\frac{1}{S} \int_{0}^{1}\left(\nabla^{2} \psi\right)^{2} r d r
$$

where we have invoked the boundary conditions $\partial \psi / \partial r=0$ at $r=0$ and $r=1$. The right hand side of equation (21) is simply the rate of Ohmic heating. By setting $\partial \psi / \partial r=0$ at $r=1$, we ensure that the Poynting flux is locally zero at all points on the boundary. We are not setting $v=0$ at $r=1$, and therefore we are allowing the possibility of a local mass flow through the boundary. However, since the radial component of the velocity vector, like the equilibrium field, has a $\cos 2 \theta$ dependence (eq. [12]), the integrated mass flux through the boundary is always zero. Thus, after $t=0$ there is no net flow of energy into or out of the system, and the energy can only decrease, through Ohmic dissipation, with the rate of decrease determined purely by the internal dynamics of the system.

It is clear from equation (21) that the system is conservative in the limit $S \rightarrow \infty$, even when $\delta_{e}$ is finite: electron inertial effects are essentially reactive and thus do not give rise to dissipation. However, we will demonstrate that the field energy $\mathcal{E}_{\mathrm{f}}$ can change rapidly when $\delta_{e}$ is finite and $S \rightarrow \infty$. The conservation of $\mathcal{E}$ in this limit provides a critical test of the numerical schemes used for solving the full equations. In Appendix A we obtain a relationship between field energy and electron kinetic energy that will prove to be useful for the interpretation of finite $\delta_{e}$ solutions presented in $\S \S 3.4$.

\subsection{Ideal MHD Solutions}

As first noted by Bulanov \& Syrovatskii (1981), a simple analytical solution of the linearized equations exists in the ideal MHD limit $\left(S \rightarrow \infty, \delta_{e} \rightarrow 0\right)$. This solution provides an additional test of the numerical schemes, and moreover is key to understanding certain effects observed in the non-ideal case.

Following Craig \& Watson (1992), we evaluate the ideal MHD solution explicitly for two alternative initial conditions. When $\delta_{e}=1 / S=0$ equations (15) and (16) can be 
combined to give

$$
\frac{\partial^{2} \psi}{\partial t^{2}}=r \frac{\partial}{\partial r}\left(r \frac{\partial \psi}{\partial r}\right) .
$$

With the substitution $u=\ln r$, equation (22) reduces to the one dimensional wave equation

$$
\frac{\partial^{2} \psi}{\partial t^{2}}=\frac{\partial^{2} \psi}{\partial u^{2}}
$$

with general solution

$$
\psi=f(u+t)+g(u-t)=f(\ln r+t)+g(\ln r-t),
$$

the functions $f$ and $g$ being arbitrary. Employing the boundary conditions $\partial \psi / \partial r=0$ at $r=0$ and $r=1$ and initial conditions

$$
\frac{\partial \psi}{\partial r}=\sin (\pi r),
$$

it is straightforward to show that $f$ and $g$ are such that the full solution is given by

$$
\begin{gathered}
\psi=-\frac{1}{2 \pi}\left\{\cos \left(\pi r e^{t}\right)+\cos \left(\pi r e^{-t}\right)\right\}, t<-\ln r \\
\psi=-\frac{1}{2 \pi}\left\{\cos \left(\frac{\pi}{r} e^{-t}\right)+\cos \left(\pi r e^{-t}\right)\right\}, t>-\ln r
\end{gathered}
$$

Putting $\delta_{e}=1 / S=0$ in equation (15), we obtain

$$
\begin{gathered}
v=\frac{1}{2}\left\{e^{t} \sin \left(\pi r e^{t}\right)-e^{-t} \sin \left(\pi r e^{-t}\right)\right\}, t<-\ln r \\
v=-\frac{1}{2}\left\{\frac{e^{-t}}{r^{2}} \sin \left(\frac{\pi}{r} e^{-t}\right)+e^{-t} \sin \left(\pi r e^{-t}\right)\right\}, t>-\ln r
\end{gathered}
$$

The solutions for $t>-\ln r$ represent smooth continuations of those for $t<-\ln r$ : there is no discontinuity at this point. Using these expressions, we can compute the time evolution of the ion kinetic and field energy. As shown in the left plot of Figure 1, the two components of the energy undergo a single oscillation before reaching equipartition in about three Alfvén times. The field and velocity profiles continue to evolve after this time, however. As shown in the right hand frame of Figure 1, the field energy and kinetic energy become increasingly concentrated in the vicinity of the X-point. Eventually, the field and velocity gradients become sufficiently large that the neglect of resistive and electron inertial terms in equations (15) and (16) is no longer justified and the ideal MHD solution becomes invalid. The right hand frame of Figure 1 exemplifies the well-known tendency of magnetic X-points to focus and accrete electromagnetic and kinetic energy (see e.g. Craig \& Watson 1992). 
The trajectory of the inward-propagating wave described by equation (24) is given by $d r / d t=-r$ : this arises from the Alfvénic character of the wave and the fact that the magnitude of the equilibrium magnetic field is proportional to $r$ (cf. eq. [7]). McLaughlin \& Hood (2004) have recently demonstrated an important consequence of this, namely that a disturbance initially consisting of a plane wave is refracted as it approaches the $\mathrm{X}$-point in such a way that it becomes more azimuthally symmetric: the region of the wave front closest to the null propagates more slowly than neighboring regions. For this reason, it is particularly appropriate to consider the evolution of azimuthally symmetric perturbations.

It is also instructive to compute the ideal MHD solution for the case of a more localized initial flux perturbation of the form

$$
\psi=-\frac{\Delta u^{2}}{2} \exp \left[-\left(\frac{\ln r}{\Delta u}\right)^{2}\right],
$$

where $\Delta u$ is a constant characterizing the width of the disturbance in $\ln r$ space. Craig \& Watson (1992) considered initial perturbations similar to this, but with a factor of $\ln r$ outside the exponential (so that $\psi$ rather than $\partial \psi / \partial r$ was set equal to zero at $r=1$ ) and $\Delta u=1$. Profiles of this type are only strongly localized if $\Delta u \ll 1$. Determining $\psi(r, t)$ for this case from equation (24), and using equation (15) with $\delta_{e}=1 / S=0$ to compute $v$, we obtain

$$
\begin{gathered}
\frac{\partial \psi}{\partial r}=\frac{1}{2 r}\left\{(\ln r+t) \exp \left[-\left(\frac{\ln r+t}{\Delta u}\right)^{2}\right]+(\ln r-t) \exp \left[-\left(\frac{\ln r-t}{\Delta u}\right)^{2}\right]\right\}, \\
v=\frac{1}{2 r}\left\{(\ln r+t) \exp \left[-\left(\frac{\ln r+t}{\Delta u}\right)^{2}\right]-(\ln r-t) \exp \left[-\left(\frac{\ln r-t}{\Delta u}\right)^{2}\right]\right\} .
\end{gathered}
$$

If $\Delta u \ll 1, \partial \psi / \partial r$ and $v$ are generally negligible except for $\ln r \sim \pm t$. Since we are only considering $t>0$ and $r<1$, i.e. $\ln r<0$, the dominant terms in equation (29) are those multiplied by $\ln r+t$, representing an inward propagating wave. It is immediately apparent in this case that $v \simeq \partial \psi / \partial r$, i.e. energy is equally partitioned between the non-potential component of the magnetic field and the bulk flow, as in the case of the global disturbance represented by equation (25).

The energy components corresponding to this solution are plotted versus time for $\Delta u=0.1$ in the left hand frame of Figure 2. It is apparent that equipartition occurs much more rapidly than in the case of Figure 1. The right hand frame of Figure 2, showing the evolution of $\partial \psi / \partial r$, indicates as before rapid focusing of the field energy. Steepening of the profiles is noticeably more rapid than in the case of Fig. 1: we would accordingly expect non-ideal effects to occur at an earlier stage of the system evolution. The two solutions considered above illustrate the fact that energy equipartition is a natural asymptotic state of the ideal MHD system (cf. Craig \& Watson 1992). 


\subsection{Resistive MHD Solutions}

In this subsection we present results obtained from numerical solutions of equations (15) and (16) for the resistive MHD case, $\delta_{e}=0$. This scenario, with somewhat different initial conditions, was investigated extensively by Craig \& Watson (1992): we consider it here in order to provide an exact benchmark for the finite $\delta_{e}$ cases considered in $\S \S 3.4$, and also to highlight a phase in the late evolution of $\mathcal{E}$ not discussed by those authors. Independently-written codes were used to solve equations (15) and (16) for $\delta_{e}=0$ and for finite $\delta_{e}(\S \S 3.4)$. The numerical methods used in these codes are described briefly in Appendix B.

Our initial conditions are those defined by equations (25) and (28), together with $v=0$, as before. In the case of the more localized field perturbation (eq. [28]), we take $\Delta u=0.1$. For the two types of initial field perturbation, we have determined the evolution of the system for a wide range of Lundquist numbers $S$, from 10 to $10^{8}$; Craig \& Watson (1992) concentrated their investigation on the case of $S=10^{8}$. Our results, shown in Figures 3 and 4, concur at high $S$ with those obtained by Craig \& Watson. For $S>10^{3}$ there is an early phase in which the system evolution is well-described by ideal MHD. In Figure 3(h), for example $\left(S=10^{8}\right)$, the time evolution of the energy components up to $t \simeq 7$ is identical to that computed using the corresponding ideal solution (Figure 1). The ideal phase becomes progressively longer as $S$ is increased: for very small $S$, on the other hand, there is no ideal phase and the energy decays immediately. Although the onset time of the drop in energy increases with $S$, the timescale of the decay itself is essentially independent of this parameter, and is comparable to or less than one Alfvén time. The factor by which the energy falls at this point appears to depend critically on the initial field perturbation: this was also found by Craig \& Watson. In the case of the more localized perturbation (Fig. 4), the energy is reduced in this phase by a factor of about $e^{10} \simeq 2 \times 10^{4}$.

The rapid drop in energy is followed by a period of slower decay in which the field energy and kinetic energy exhibit oscillatory behavior. As noted by Craig \& Watson (1992), these oscillations arise from the discrete spectrum of weakly damped eigenmodes studied by Craig \& McClymont (1991). After a sufficiently long period, the oscillations disappear. This can be seen in Figures 3 and 4 for $S \leq 10^{3}$, and in Figure 5 for $S=10^{4}$, $10^{5}$. The oscillations persist for a time that increases as $(\ln S)^{2}$ : this is consistent with the $S$ dependence of the least damped discrete mode (eq. [1]).

After the oscillatory phase, the energy continues to decay, but on a longer timescale, and the energy moreover is almost entirely kinetic $\left(\mathcal{E}_{\mathrm{ki}} \gg \mathcal{E}_{\mathrm{f}}\right)$. This late phase of nonoscillatory decay, which is apparent in Figures 3(a), 3(b), 4(a), 4(b) and 5, can be accounted for by the fact that the discrete modes identified by Craig \& McClymont (1991) do not constitute a complete set: as demonstrated by McClements \& Thyagaraja (2004), there is also a continuum of damped modes with zero real frequency. When $\delta_{e}$ 
and $S$ are finite, equation (22) is replaced with the fourth order equation

$$
\ddot{\psi}-\frac{\delta_{e}^{2}}{r} \frac{\partial}{\partial r}\left(r \frac{\partial \ddot{\psi}}{\partial r}\right)=r \frac{\partial}{\partial r}\left(r \frac{\partial \psi}{\partial r}\right)+\frac{1}{S r} \frac{\partial}{\partial r}\left(r \frac{\partial \dot{\psi}}{\partial r}\right) .
$$

For $\psi=e^{-i \omega t} f(r)$, equation (30) reduces to

$$
\omega^{2} r f+\left(r^{2}-\omega^{2} \delta_{e}^{2}-i \frac{\omega}{S}\right) \frac{d}{d r}\left(r \frac{d f}{d r}\right)=0 .
$$

The coefficient of the highest order derivative in this equation vanishes for complex mode frequencies $\omega$ satisfying

$$
\omega^{2} \delta_{e}^{2}+i \frac{\omega}{S}=r^{2}
$$

McClements \& Thyagaraja showed that this relation gives rise to two distinct continua: one with finite real frequency, the other with zero real frequency. The former, which we will discuss in the next section, only exists when $\delta_{e} \neq 0$. The zero real frequency continuum, on the other hand, exists whether $\delta_{e}$ is finite or not. This can be seen by putting $\omega=-i \gamma$, where $\gamma$ is taken to be real. Equation (32) then yields

$$
\gamma=\frac{1}{2 S \delta_{e}^{2}}\left[1 \pm\left(1-4 r^{2} S^{2} \delta_{e}^{2}\right)^{1 / 2}\right]
$$

In the limit $S^{2} \delta_{e}^{2} \ll 1$, the negative root in this expression yields a damping rate

$$
\gamma=S r^{2}
$$

Thus, there is a purely damped continuum mode for each point in the solution domain. Since this extends to $r=0$, the damping can be arbitrarily weak. In the resistive MHD case, the Fourier spectra of $\psi$ and $v$ will include contributions from this continuum as well as the discrete modes identified by Craig \& McClymont. Equation (34) indicates that non-potential perturbations to the X-point field will in general take an infinite time to be completely dissipated. The presence of the continuum only becomes apparent when the discrete modes have disappeared: since the discrete mode damping rate scales with $1 /(\ln S)^{2}$ (eq. [1]), the presence of the continuum becomes manifest at progressively later times as $S$ is increased.

As noted above, another feature of the late non-oscillatory decay phase is that the remaining energy in this period is almost entirely kinetic. This can be understood qualitatively by putting $\omega=-i \gamma$ and writing equation (31) in the form

$$
\left(r f^{\prime}\right)^{\prime}=\frac{\gamma^{2} r f}{r^{2}-\gamma / S}
$$

where primes denote derivatives with respect to $r$. Assuming that $f$ tends to a finite value as $r$ approaches the singular point $(\gamma / S)^{1 / 2}$, as in the case of the finite 
real frequency continuum modes discussed by McClements \& Thyagaraja (2004), we infer from equation (35) that whereas the magnetic field perturbation $f^{\prime}$ diverges logarithmically in the neighborhood of $r=(\gamma / S)^{1 / 2}$, the current $\left(r f^{\prime}\right)^{\prime} / r$ and hence the velocity (cf. eq. [16]) diverge as $1 /\left[r-(\gamma / S)^{1 / 2}\right]$. For these continuum eigenfunctions, the field energy is thus square integrable whereas the kinetic energy is not. The energy components in the late non-oscillatory phase of the resistive MHD simulations can, in principle, be synthesized using these singular eigenmodes. In view of the nature of the singularities in $f^{\prime}$ and $\left(r f^{\prime}\right)^{\prime} / r$, it is not surprising that the kinetic energy, although necessarily finite (as indicated by eq. [21], the total energy can never increase), is large compared to the field energy.

\subsection{Finite Collisionless Skin Depth Solutions}

We have obtained numerical solutions of equations (15) and (16) with $\delta_{e}=0.01$ and $S$ ranging from $10^{2}$ to $10^{6}$, using both types of initial condition discussed in the previous two subsections. Figure 6 shows the evolution of the field energy, ion kinetic energy and electron kinetic energy in each case, with the left hand and right hand plots corresponding respectively to the spatially extended initial condition (eq. [25]) and the strongly localized initial condition (eq. [28]). Comparing Figure 6 with Figures 3 and 4 , it can be seen that electron inertia has a negligible impact on the evolution of $\mathcal{E}_{\mathrm{f}}$, $\mathcal{E}_{\text {ki }}$ and $\mathcal{E}$ whenever $S \delta_{e}^{2}<1$ : the black, red and blue curves in Figure 3(b) and the left hand frame of Figure 6(a), for example, which were obtained using the same initial conditions and $S=10^{2}$, are indistinguishable. This is particularly noteworthy since the results shown in Figure 6 and those discussed in $\S \S 3.3$ were obtained using two independent codes (see Appendix B). When $S \delta_{e}^{2}=0.01, \mathcal{E}_{\mathrm{ke}}$ remains below the other energy components at all times for both sets of initial conditions (Fig. 6[a]). When the initial field perturbation has the profile $\sin \pi r$, the ratio $\mathcal{E}_{\text {ke }} / \mathcal{E}_{\mathrm{f}}$ is well-approximated at $t=0$ by the equals sign in equation (A7). The ratio is somewhat higher in the case of the more localized initial field perturbation: this appears to be due to a greater

contribution of eigenvalues $\lambda_{n}^{2}>\lambda_{0}^{2}$ in equation (A8). Towards the end of simulations with $S \delta_{e}^{2}=0.01, \mathcal{E}_{\mathrm{ke}}$ becomes comparable to the field energy, but the system evolution is still well-approximated by resistive MHD.

The situation is completely different when $S \delta_{e}^{2}>1$ : this regime is represented by Figures $6(\mathrm{~d})$ and $6(\mathrm{e})$, with Figure $6(\mathrm{c})$ representing a transitional case $\left(S \delta_{e}^{2}=1\right)$. The oscillatory phase prevalent in the resistive MHD simulations is still present when $S \delta_{e}^{2}=1$ but wholly absent when this quantity is significantly greater than unity. After a few Alfvén times, the total energy is dominated by ions and electrons, with $\mathcal{E}$ equally partitioned between the two species. The total energy still decays, as it must according to equation (21), but at a diminishing rate as $S$ increases. In fact, the asymptotic decay time of the total energy is equal to $S \delta_{e}^{2}$. Given that $\mathcal{E}$ is dominated by electrons and ions, with $\mathcal{E}_{\mathrm{ki}} \simeq \mathcal{E}_{\mathrm{ke}}$ so that $\mathcal{E}_{\mathrm{ke}} \simeq \mathcal{E} / 2$, the decay rate follows very simply from equation 
$(21)$ :

$$
\frac{d \mathcal{E}}{d t}=-\frac{1}{S \delta_{e}^{2}} \int_{0}^{1} \delta_{e}^{2}\left(\nabla^{2} \psi\right)^{2} r d r=-\frac{2 \mathcal{E}_{\mathrm{ke}}}{S \delta_{e}^{2}} \simeq-\frac{\mathcal{E}}{S \delta_{e}^{2}}
$$

McClements \& Thyagaraja (2004) demonstrated that the discrete X-point spectrum ceases to exist when the intrinsic damping rate of the finite frequency continuum is less than that of the least damped discrete mode. As noted previously, the latter varies as $1 /(\ln S)^{2}$ and is of order unity in Alfvén units for low $S$. Thus, when $S \delta_{e}^{2} \gg 1$ the spectrum is purely continuous. The decay rate indicated by equation (36) is exactly twice the intrinsic damping rate of finite real frequency continuum eigenmodes (cf. eq. [3]). This is to be expected if the energy of the system is assumed to lie predominately in the finite frequency continuum, since the ion and electron kinetic energies scale as the squares of, respectively, the fluid velocity and the current. As noted in $\S 1$, the energy decay time $S \delta_{e}^{2}$ is simply the electron collision time if $S$ is determined by Spitzer resistivity.

For these simulations there is a period in which the field energy decays much more rapidly than this, typically on an Alfvén timescale: this causes $\mathcal{E}_{\mathrm{f}}$ to be energetically insignificant at later times. As in the later stages of the resistive MHD simulations, the dominance of kinetic energy over field energy when $S \delta_{e}^{2}>1$ is associated with the fact that for continuum eigenfunctions the singularity in the field is square integrable while those in the current and velocity are not (McClements \& Thyagaraja 2004). The only difference in this case is that part of the continuum has finite real frequency.

The field energy decays on the Alfvén timescale even in the collisionless limit $\left(S \delta_{e}^{2} \rightarrow\right.$ $\infty)$ : we thus observe irreversible behavior, despite the fact that total energy is conserved in this case. As demonstrated by McClements \& Thyagaraja (2004), the spectrum in the limit $S \delta_{e}^{2} \rightarrow \infty$ consists of a band-limited continuum. The decay of field energy in this limit can be interpreted as continuum damping. It is exactly analogous to Landau damping: the Vlasov-Poisson system of equations, like the system represented by equations (15) and (16) in the limit $S \delta_{e}^{2} \rightarrow \infty$, is conservative but exhibits irreversible behavior (see e.g. van Kampen \& Felderhof 1967). The analysis of inviscid plane Couette flow by Case (1960) shows that this type of behavior is not restricted to plasma kinetic theory or wave-particle interactions but is a general consequence of phase mixing. Thyagaraja et al. (2002) have demonstrated that even the simplest advection-diffusion equation in one space dimension can exhibit such dissipationless damping. In the solar context, phase mixing of MHD waves has been investigated by many authors as a possible coronal heating mechanism (e.g. Heyvaerts \& Priest 1983; Voitenko \& Goosens 2000; Tsiklauri, Nakariakov, \& Rowlands 2003). Porcelli et al. (2002) have shown that the channeling of field energy into kinetic energy through phase mixing occurs in the collisionless evolution of magnetic islands. In the case of finite $S$ with $S \delta_{e}^{2}>1$, as in Figures $6(\mathrm{~d})$ and $6(\mathrm{e})$, the field energy decays through a combination of phase mixing and the intrinsic finite frequency continuum mode damping given by equation (3). 
Another characteristic of the $S \delta_{e}^{2}>1$ regime indicated by Figure 6 is that the evolution of $\mathcal{E}$ is relatively insensitive to the initial conditions: in Figures $6(\mathrm{~d})$ and $6(\mathrm{e})$, with $S \delta_{e}^{2}$ equal to 10 and 100 respectively, the left hand plots are almost identical to the right hand plots. In the case of the more localized perturbation, the electrons and ions take somewhat less time to reach equipartition, but the subsequent evolution of the energy components is essentially independent of the initial field profile. In the resistive MHD regime, in contrast, it is clear from Figures 3 and 4 that the total decrement in the energy after a given time interval depends critically on the initial conditions, although the rate of energy loss does not.

The profiles of $\partial \psi / \partial r$ and $v$ also depend critically on whether $S \delta_{e}^{2}$ is less than or greater than unity. Figure 7 shows the evolving velocity profile up to $t=4$ for the case of initial $\psi \propto-\exp \left[-(\ln r)^{2} / \Delta u^{2}\right], \delta_{e}=0.01$ and (a) $S=10^{3}$, (b) $S=10^{5}$. For $S \delta_{e}^{2}<1$, the profile steepening observed in the ideal phase (Figs. [1] and [2]) ceases due to resistive effects, and the field and velocity are damped without the shapes of their spatial profiles being radically altered. A low amplitude ripple appears in the wake of the inward propagating pulse, apparently due to finite $\delta_{e}$ (the ripple is absent in the resistive MHD limit), but, as indicated by Figure 8(a), the overall profile shape is similar to the ideal MHD profiles shown in the right hand frame of Figure 2. When $S \delta_{e}^{2}>1$, on the other hand, the field and velocity profiles are characterized by short wavelength features that persist for long times (Figs. 7[b] and 8[b]). Porcelli et al. (2002) observed a similar filamentation process in collisionless simulations of a magnetic island. The appearance of progressively more nodes in the field profile means that the Rayleigh quotient defined by equation (A2) increases with time: this is linked to the rapid decay of field energy relative to electron kinetic energy noted above. Figures 7(b) and 8(b) indicate that the radial wave number spectrum is cascading into progressively shorter wavelengths. Examples of such direct cascades investigated previously in the plasma literature (e.g. by Thyagaraja, Loureiro, \& Knight 2002) show that they play a crucial role in the temporal evolution of field energy.

The occurrence and persistence of small spatial scales for $S \delta_{e}^{2}>1$ is linked to the fact that the only eigenmodes in this limit are singular, and are thus characterized by arbitrarily short wavelengths: the energy in the initial perturbation is channeled into these short wavelengths, giving rise to the observed profiles. McClements \& Thyagaraja (2004) showed that the finite frequency continuum is present even when $S \delta_{e}^{2}<1$, but in this case the component of the energy in this continuum rapidly damps out (on the $S \delta_{e}^{2}$ timescale), and the remaining energy decays on a timescale determined by the discrete mode damping rate. Since the discrete modes are non-singular, they are not characterised by short wavelengths. Thus, in the resistive MHD regime we observe relatively smooth spatial profiles (Figs. 7[a] and 8[a]) but highly structured evolution of the total energy (Figs. 3, 4, 6[a] and 6[b]), whereas in the $S \delta_{e}^{2}>1$ regime the profiles have fine scale structure (Figs. $7[\mathrm{~b}]$ and $8[\mathrm{~b}]$ ) but the temporal behavior of the total energy is relatively smooth (Figs. 6[d] and 6[e]). In the latter case short wavelength structures also appear in the current profile and the longitudinal component 
of the electric field, $-\partial \psi / \partial t$. Electrons (and ions) would then be accelerated in nested cylindrical shells, centered on the X-line, with acceleration occurring in opposite directions in adjacent shells. One attractive feature of this scenario is that it suggests the possibility of producing large numbers of hard X-ray emitting electrons without the creation of large net beam currents, and hence unacceptably large magnetic fields (see e.g. Holman 1985).

McClements \& Thyagaraja (2004) noted that the discrete X-point spectrum found by Craig \& McClymont (1991) is replaced in the ideal limit with a continuum: the eigenmodes of this continuum, like those of the non-ideal continua discussed above, are singular. The cascading of field and kinetic energy into small spatial scales apparent in Figures 1 and 2 is associated with the singular nature of the ideal MHD eigenmodes. When $S$ is finite but $\delta_{e}=0$, the finite frequency ideal MHD spectrum becomes discrete and the eigenmodes non-singular. However, as discussed in $\S \S 3.3$, the system evolution at high $S$ is still accurately described by ideal MHD for several Alfvén periods after $t=0$ : energy cascades into small scales in this period, despite the resolution of the continuum into discrete modes. Similarly, although one would expect the non-ideal continua that exist for finite $S$ and $\delta_{e}$ to be resolved into discrete spectra by, for example, finite gyro radius effects, such effects will not necessarily prevent filamentation or the decay of field energy relative to kinetic energy. On the other hand, gyro radius effects are likely to impose a lower limit on the length scale of the filaments: in the simulations reported here with $S \delta_{e}^{2}>1$, there appears to be no such lower limit.

\section{Summary and Application to Solar Flares}

We have studied the relaxation of non-potential perturbations to a current-free magnetic X-point, taking into account the effects of resistivity and electron inertia. The latter has been shown to have a negligible effect on the evolution of the system whenever the collisionless skin depth is less than the resistive scale length. Non-potential magnetic field energy in this resistive MHD limit initially reaches equipartition with flow energy, in accordance with analytical results obtained using ideal MHD, and is then dissipated extremely rapidly, on an Alfvénic timescale that is essentially independent of Lundquist number, with the energy being attenuated to a greater extent if the initial perturbation is highly localized. Following this period of rapid dissipation in the resistive case, the magnetic field energy and kinetic energy decay on a longer timescale and exhibit oscillatory behavior: Craig \& Watson (1992), who also observed such behaviour in resistive MHD X-point simulations, noted that it arises from the existence of discrete normal modes with finite real frequency. Eventually, the oscillations disappear, and the energy continues to decay on a yet longer timescale. This nonoscillatory decay arises from the fact that the discrete modes observed in the earlier evolution do not constitute a complete set, even in the resistive limit; the spectrum also contains a purely damped continuum. When the collisionless skin depth exceeds 
the resistive scale length, the system again evolves initially according to ideal MHD. At the end of this ideal phase, the field energy decays typically on an Alfvénic timescale, while the kinetic energy (which is equally partitioned between ions and electrons in this case) is dissipated on the electron collision timescale. The oscillatory decay in the energy observed in the resistive case is absent, but short wavelength structures appear in the field and velocity profiles, suggesting the possibility of particle acceleration in oppositely-directed current channels.

We now consider the possible application of these results to the problem of short timescale energy release in solar flares. For a system size $R_{0}$ equal to the typical length of a flaring coronal loop $\left(\sim 10^{7} \mathrm{~m}\right), B_{0} \sim 0.1 \mathrm{~T}, n=10^{15} \mathrm{~m}^{-3}, T \sim 3 \times 10^{6} \mathrm{~K}$, and Spitzer resistivity, the Lundquist number $S$ is of the order of $3 \times 10^{15}$ while the dimensionless collisionless skin depth is about $10^{-8}$ and hence $S \delta_{e}^{2} \simeq 1$. This indicates that the collisionless skin depth $\delta_{e}$, although very small compared to the typical spatial extent of a solar flare, is actually comparable to the resistive length scale $1 / S^{1 / 2}$ if the latter is assumed to be determined by Coulomb collisions. Since Spitzer resistivity is often invoked as the source of magnetic field energy dissipation in flares, the figures quoted above suggest that electron inertial effects are likely to play a role in this process. However, in the absence of precise knowledge of magnetic field structures in the pre-flare corona, there is no particular reason for identifying $R_{0}$ with a macroscopic flare dimension, given that no other length scales appear in the current-free X-point field defined by equation (7). Indeed, it is unlikely that this equation would be a good approximation to the coronal field over length scales as large as $10^{7} \mathrm{~m}$. More realistically, it could be regarded as a model of a spatially restricted region, with dimensions much smaller than a typical flare size, for which the approximation of a current-free twodimensional X-point is appropriate. Non-potential magnetic field energy originating from a much more extensive region could still be channeled into the X-point through the focusing effect noted by Craig \& Watson (1992). A key point to note here is that $S$ is defined in terms of a field component which increases linearly with distance from a two-dimensional null (it is independent of any longitudinal field component). If the boundary is chosen to be arbitrarily close to the origin, the Lundquist number is then arbitrarily small. In this specific context, it is thus entirely appropriate to consider values of $S$ much smaller than the global Lundquist numbers characteristic of the flaring solar corona, and values of $\delta_{e}$ much larger than the figure of $10^{-8}$ quoted above.

The choice of $R_{0}$ is not completely arbitrary, since the boundary conditions imposed at this radius must have some effect on the evolution of $\mathcal{E}$. However, the results presented in the previous section and by Craig \& Watson (1992) suggest strongly that any such effect is very weak. In the resistive regime, our results are essentially identical to those obtained by Craig \& Watson despite the use of different boundary conditions. This is consistent with the fact that the time-evolving field and velocity profiles in both the resistive MHD and electron inertial regimes invariably show dissipation occurring much closer to the $\mathrm{X}$-point than the boundary at $R=R_{0}$. 
Regardless of the initial conditions, our results show the greater part of the nonpotential magnetic field energy either being dissipated or, in the case of $S \delta_{e}^{2}>1$, being converted into kinetic energy, on the Alfvén time $\tau_{A}=R_{0} / c_{A 0}$. In the event of $S \delta_{e}^{2}$ being greater than unity, with $S$ determined by Spitzer resistivity, the kinetic energy is dissipated on the electron collision time $\tau_{e}$. Since $S \delta_{e}^{2}$ is simply the ratio $\tau_{e} / \tau_{A}$ (cf. eqs. [3] and [4]), the overall energy dissipation timescale can thus be written as $\max \left(\tau_{A}, \tau_{e}\right)$. As noted in $\S 1, \tau_{e}$ is typically less than a second in the flaring corona. The Alfvén time could also be less than a second, if the X-point field were sufficiently highly sheared. Again putting $n=10^{15} \mathrm{~m}^{-3}$, and assuming that the field rises to, say, $0.01 \mathrm{~T}$ at a radial distance from the null of $10^{6} \mathrm{~m}$, we obtain $\tau_{A} \simeq 0.1 \mathrm{~s}$. Since it is the destruction of magnetic flux that gives rise to an accelerating electric field in the $z$ direction $E_{z}=-\partial \psi / \partial t$, the key timescale from the point of view of energetic particle production is always $\tau_{A}$, provided that the acceleration time is no longer than this. The latter timescale depends, of course, on the amplitude of the initial magnetic field perturbation. Since equations (15) and (16) are linear, the perturbation amplitude is arbitrary as far as the results shown in Figures 1-8 are concerned. However, we can at least check that the quantity of magnetic flux $\Delta \psi$ that must be destroyed to produce, say, a $30 \mathrm{keV}$ electron in $0.1 \mathrm{~s}$ is broadly consistent with the linear approximation. The energy $\mathcal{E}_{e}$ acquired by a nonrelativistic electron in time $t$ is

$$
\mathcal{E}_{e} \sim \frac{e^{2} t^{2}}{2 m} \frac{\Delta \psi^{2}}{\tau_{A}^{2}},
$$

so that

$$
\Delta \psi=\left(2 m \mathcal{E}_{e}\right)^{1 / 2} \frac{\tau_{A}}{e t} .
$$

The perturbed field is $\tilde{B}_{\varphi} \sim \Delta \psi / R_{0}$ and the unperturbed field is $B_{E} \sim B_{0}$ : their ratio is

$$
\frac{\tilde{B}_{\varphi}}{B_{E}} \sim\left(2 m \mathcal{E}_{e}\right)^{1 / 2} \frac{\tau_{A}}{e t R_{0} B_{0}}
$$

Using the parameter values invoked above, we obtain $\tilde{B}_{\varphi} / B_{E} \sim 10^{-7}$. Even allowing for steepening of the perturbed field during the course of each simulation, this crude estimate suggests that the production of $30 \mathrm{keV}$ electrons on sub-second timescales is easily compatible with the use of a linear model.

We have omitted from our model many effects that could in principle influence the field decay time. These include ion gyro radius effects, equilibrium currents and flows, longitudinal magnetic fields, Hall currents, nonlinear effects, pressure gradients, the effects of three dimensional geometry, compressibility and kinetic effects. For example, both McClymont \& Craig (1996) and Biskamp et al. (1997), using very different physical models, have found that the reconnection rate can be strongly affected by the presence of a longitudinal magnetic field. Our purpose in this paper has been to achieve a reasonably complete understanding of a relatively simple physical model, with the minimum number of free parameters. To achieve this understanding, we have 
made full use of the spectral analysis presented in our earlier paper (McClements \& Thyagaraja 2004), although the complementary initial value approach was essential in order to demonstrate some of our key results, notably collisionless continuum damping of the field energy on the Alfvén timescale. The scheme we have developed could be readily extended to incorporate most of the additional effects listed above. The results in this paper demonstrate that resistivity and electron inertia alone are sufficient to endow perturbed magnetic X-points with a rich physical structure.

This work was funded by the United Kingdom Engineering and Physical Sciences Research Council. Helpful conversations with Per Helander are gratefully acknowledged.

\section{Appendix A: Relation between Electron Kinetic Energy and Field Energy}

We can establish a useful relation between the field energy $\mathcal{E}_{\mathrm{f}}$ and the electron kinetic energy $\mathcal{E}_{\text {ke }}$ as follows. The latter is given by

$$
\mathcal{E}_{\mathrm{ke}}=\frac{1}{2} \delta_{e}^{2} \int_{0}^{1} \frac{1}{r}\left[\frac{\partial}{\partial r}(r \varphi)\right]^{2} d r
$$

where $\varphi=-\partial \psi / \partial r$ is the (purely azimuthal) perturbation to the magnetic field. This expression prompts us to consider the Rayleigh quotient $R[\varphi]$ defined by the expression

$$
R[\varphi]=\frac{\int_{0}^{1} \frac{1}{r}\left[\frac{\partial}{\partial r}(r \varphi)\right]^{2} d r}{\int_{0}^{1} \varphi^{2} r d r}
$$

Following the ideas underlying Wirtinger's inequality (Hardy, Littlewood, \& Polya 1967), we attempt to minimize this functional over all test functions $\varphi$ that vanish at $r=0$ and $r=1$. According to the Rayleigh-Ritz principle frequently employed in wave mechanics (e.g. Mathews \& Walker 1964), the minimum is attained for $\varphi$ satisfying the Euler-Lagrange equation

$$
\frac{\partial}{\partial r}\left[\frac{1}{r} \frac{\partial}{\partial r}(r \varphi)\right]+\lambda_{0}^{2} \varphi=0,
$$

where $\lambda_{0}^{2}>0$ is the smallest eigenvalue of this equation. Denoting the corresponding eigenfunction by $\varphi_{\min }$, it is straightforward to establish that

$$
\varphi_{\min }=A J_{1}\left(\lambda_{0} r\right)
$$

where $J_{1}$ is the Bessel function of order one and $A$ is a normalization constant that can be chosen to be unity without loss of generality. Evidently $\lambda_{0}$ is equal to the 
first positive zero of $J_{1}$, i.e. $j_{1,1} \simeq 3.8$ (e.g. Abramowitz \& Stegun 1965). From equations (A2) and (A3), integrating the numerator of the former by parts and using the boundary conditions $\varphi=0$ at $r=0$ and $r=1$, we infer the inequality

$$
R[\varphi] \geq \lambda_{0}^{2}=j_{1,1}^{2} \simeq 14.4 .
$$

The equals sign in this result applies if and only if $\varphi=\varphi_{\min }=J_{1}\left(\lambda_{0} r\right)$. Since the field energy is given by

$$
\mathcal{E}_{\mathrm{f}}=\frac{1}{2} \int_{0}^{1}\left(\frac{\partial \psi}{\partial r}\right)^{2} r d r=\frac{1}{2} \int_{0}^{1} \varphi^{2} r d r,
$$

it follows that $R[\varphi]=\mathcal{E}_{\mathrm{ke}} /\left(\mathcal{E}_{\mathrm{f}} \delta_{e}^{2}\right)$ and hence

$$
\mathcal{E}_{\mathrm{ke}} \geq \lambda_{0}^{2} \delta_{e}^{2} \mathcal{E}_{\mathrm{f}} .
$$

Thus, for any perturbation to the equilibrium X-point magnetic field, the electrons must have finite kinetic energy at all times.

In general, $\varphi$ can be expanded in a Fourier-Bessel series of the form

$$
\varphi=\sum_{n=0}^{\infty} \sqrt{2} a_{n} J_{1}\left(\lambda_{n} r\right) / J_{2}\left(\lambda_{n}\right),
$$

where $\lambda_{n}$ is the $(n+1)$-th positive zero of $J_{1}$, the $a_{n}$ are constants, and we have used the relation (Whittaker \& Watson 1927)

$$
\int_{0}^{1} J_{1}\left(\lambda_{n} r\right) J_{1}\left(\lambda_{m} r\right) r d r=J_{2}^{2}\left(\lambda_{n}\right) \delta_{m n} / 2,
$$

$J_{2}$ being the Bessel function of order two and $\delta_{m n}$ the Kronecker delta symbol, to ensure that the eigenfunctions form a complete orthonormal set. The Rayleigh quotient is then given by

$$
R[\varphi]=\frac{\sum_{n=0}^{\infty} a_{n}^{2} \lambda_{n}^{2}}{\sum_{n=0}^{\infty} a_{n}^{2}},
$$

i.e. a weighted mean eigenvalue of the differential operator in equation (A3), the weights in question being the squares of the Fourier-Bessel amplitudes $a_{n}$ of the perturbed field $\varphi$. Since the $\lambda_{n}$ form a monotonic increasing sequence and the index $n$ counts the number of nodes of the eigenfunction, it follows that $R$ can be regarded as a measure of the effective radial wave number of $\varphi$ (cf. Thyagaraja 1979). The initial value of this measure relates to the localization of the initial state. If $\varphi$ became more localized or developed more nodes as the system evolved, it would be characterized by relatively high eigenvalues $\lambda_{n}^{2}$, and we would then expect a relatively high ratio of electron kinetic energy to field energy. 


\section{Appendix B: Numerical Algorithms}

To solve equations (15) and (16) numerically it is convenient to introduce new variables $u=r v, b=r \partial \psi / \partial r$ and

$$
w=b-\delta_{e}^{2} r \frac{\partial}{\partial r}\left(\frac{1}{r} \frac{\partial b}{\partial r}\right) .
$$

Differentiating equation (15) with respect to $r$ and multiplying by $r$ we obtain

$$
\frac{\partial w}{\partial t}=r \frac{\partial u}{\partial r}+\frac{1}{S} r \frac{\partial}{\partial r}\left(\frac{1}{r} \frac{\partial b}{\partial r}\right),
$$

while the momentum equation becomes

$$
\frac{\partial u}{\partial t}=r \frac{\partial b}{\partial r}
$$

For the case of finite $\delta_{e}$ we solve these equations using a staggered leap-frog method, in which $u(r, t)$ is displaced with respect to $b(r, t)$ and $w(r, t)$ by half the space step, $\Delta r$. Labelling the time step by $\Delta t$, and the space and time grid points by $i$ and $n$ respectively, we approximate equations (B1) - (B3) using the finite difference scheme

$$
\begin{gathered}
\frac{u_{i+1 / 2}^{n+1}-u_{i+1 / 2}^{n}}{\Delta t}=\frac{r_{i+1 / 2}}{2 \Delta r}\left(b_{i+1}^{n+1}-b_{i}^{n+1}+b_{i+1}^{n}-b_{i}^{n}\right), \\
\frac{w_{i}^{n+1}-w_{i}^{n}}{\Delta t}=\frac{r_{i}}{2 \Delta r}\left(u_{i+1 / 2}^{n+1}-u_{i-1 / 2}^{n+1}+u_{i+1 / 2}^{n}-u_{i-1 / 2}^{n}\right) \\
+\frac{r_{i}}{S(\Delta r)^{2}}\left[\frac{b_{i+1}^{n+1}-b_{i}^{n+1}}{r_{i+1 / 2}}-\frac{b_{i}^{n+1}-b_{i-1}^{n+1}}{r_{i-1 / 2}}\right], \\
w_{i}^{n+1}=b_{i}^{n+1}-r_{i}\left(\frac{\delta_{e}}{\Delta r}\right)^{2}\left[\frac{b_{i+1}^{n+1}-b_{i}^{n+1}}{r_{i+1 / 2}}-\frac{b_{i}^{n+1}-b_{i-1}^{n+1}}{r_{i-1 / 2}}\right] .
\end{gathered}
$$

Equations (B4) - (B6) are advanced in time using an iterative predictor/corrector scheme, with a tri-diagonal matrix algorithm being used to solve equation (B6) for $b$ in terms of $w$.

The resistive solutions $\left(\delta_{e}=0\right)$ presented in $\S \S 3.3$ were obtained using a code based on a somewhat simpler technique. The discrete values of the field variable $b$ are again staggered in space with respect to those of the velocity variable $u$, but the scheme is explicit, and thus does not require iteration or matrix inversion. As noted in $\S \S 3.4$, results obtained using the two codes are essentially identical whenever $S \delta_{e}^{2}<1$. Moreover, for large $S$ and early times, both schemes recover the ideal solutions given by equations (26), (27) and (29). 


\section{References}

Abramowitz, M., \& Stegun, I. A. 1965 Handbook of Mathematical Functions (New York: Dover), 390

Birn, J., et al. 2001, J. Geophys. Res. 106, 3715

Biskamp, D., Schwarz, E., \& Drake, J. F. 1997, Phys. Plasmas, 4, 1002

Bowler, S. 2003, Astronomy \& Geophysics, 44, 6.4

Bulanov, S. V., \& Syrovatskii, S. I. 1981, Sov. J. Plasma Phys., 6, 661

Case, K. M. 1960, Phys. Fluids, 3, 143

Craig, I. J. D., \& McClymont, A. N. 1991, ApJ, 371, L41

Craig, I. J. D., \& McClymont, A. N. 1993, ApJ, 405, 207

Craig, I. J. D., \& Watson, P. G. 1992, ApJ, 393, 385

Craig, I. J. D., \& Watson, P. G. 2003, Sol. Phys., 214, 131

Hardy, G. H., Littlewood, J. E., \& Polya, G. 1967, Inequalities (Cambridge: Cambridge University Press), 184

Heyvaerts, J., \& Priest, E. R. 1983, A\&A, 117, 220

Holman, G. D. 1985, ApJ, 293, 584

Kiplinger, A. L., Dennis, B. R., Emslie, A. G., Frost, K. J., \& Orwig, L. E. 1983, ApJ, 265, L99

Lin, R. P., et al. 2002, Sol. Phys., 210, 3

Mathews, J. \& Walker, R. L. 1964, Mathematical Methods of Physics (New York: Benjamin), 315

McClements, K. G., \& Thyagaraja, A 2004, Plasma Phys. Control. Fusion, 46, 39

McClymont, A. N., \& Craig, I. J. D. 1996, ApJ, 466, 487

McLaughlin, J. A., \& Hood, A. W. 2004, A\&A, submitted

Petschek, H. E. 1964, in AAS-NASA Symposium on the Physics of Solar Flares (NASA SP-50), 425

Porcelli, F., Borgogno, D., Califano, F., Grasso, D., Ottaviani, M., \& Pegoraro, F. 2002, Plasma Phys. Control. Fusion, 44, A389

Priest, E., \& Forbes, T. 2000, Magnetic Reconnection: MHD Theory and Applications (Cambridge: Cambridge University Press)

Ramos, J. J., Porcelli, F., \& Verástegui, R. 2002, Phys. Rev. Lett., 89, 055002

Tandberg-Hanssen, E., \& Emslie, A. G. 1989, The Physics of Solar Flares (Cambridge: Cambridge University Press), 175 
Thyagaraja, A. 1979, Phys. Fluids, 22, 2093

Thyagaraja, A., Loureiro, N., \& Knight, P. J. 2002, J. Plasma Phys., 68, 363

Tsiklauri, D., Nakariakov, V. M., \& Rowlands, G. 2003, A\&A, 400, 1051

van Kampen, N. G., \& Felderhof, B. U. 1967, Theoretical Methods in Plasma Physics (Amsterdam: North Holland, 153

Voitenko, Yu., \& Goosens, M. 2000, A\&A, 357, 1073

Wesson, J. A., 1991, in Plasma Physics and Controlled Nuclear Fusion Research 1990, Vol. 2 (Vienna: International Atomic Energy Agency), 79

Whittaker, E. T., \& Watson, G. N. 1927, A Course of Modern Analysis (Cambridge: Cambridge University Press), 381 

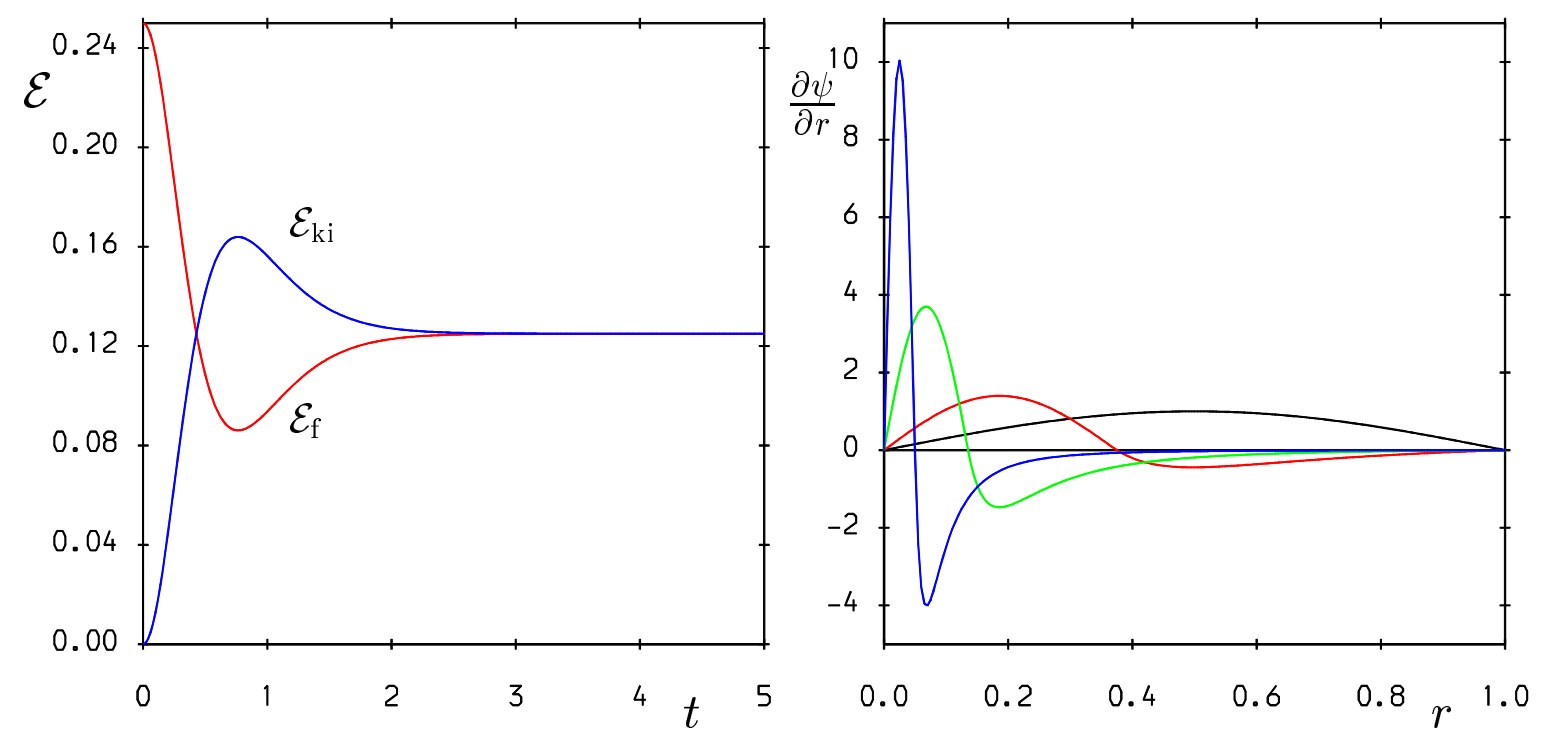

Figure 1: Left plot: time evolution of field energy (red curve) and ion kinetic energy (blue curve) in ideal MHD limit for $\partial \psi / \partial r \propto \sin (\pi r), v=0$ at $t=0$. Right plot: time evolution of $\partial \psi / \partial r$. The curves correspond to $t=0$ (black), $t=1$ (red), $t=2$ (green), and $t=3$ (blue). 

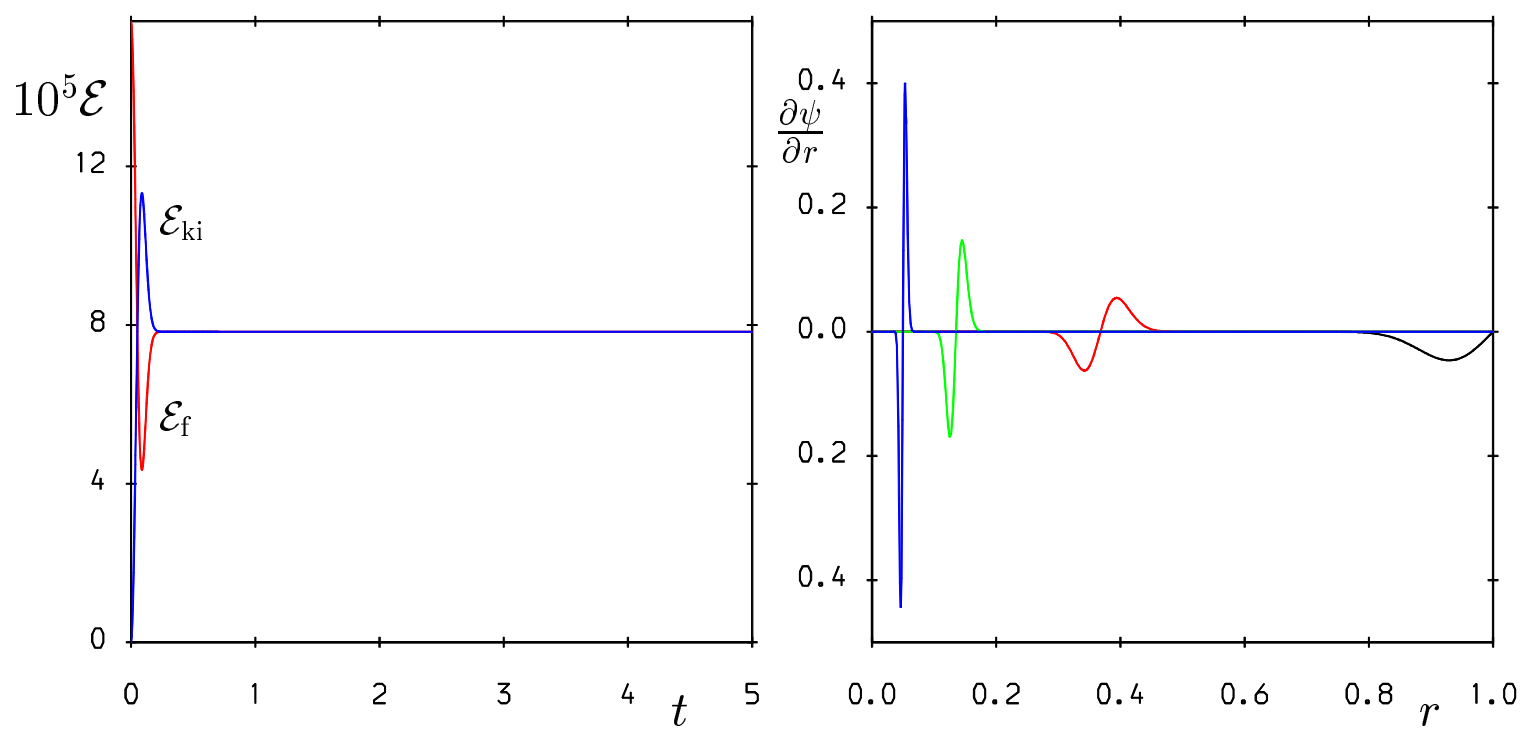

Figure 2: Left plot: time evolution of field energy (red curve) and ion kinetic energy (blue curve) in ideal MHD limit for $\psi \propto-\exp \left[-100(\ln r)^{2}\right], v=0$ at $t=0$. Right plot: time evolution of $\partial \psi / \partial r$. The curves correspond to $t=0$ (black), $t=1$ (red), $t=2$ (green), and $t=3$ (blue). 

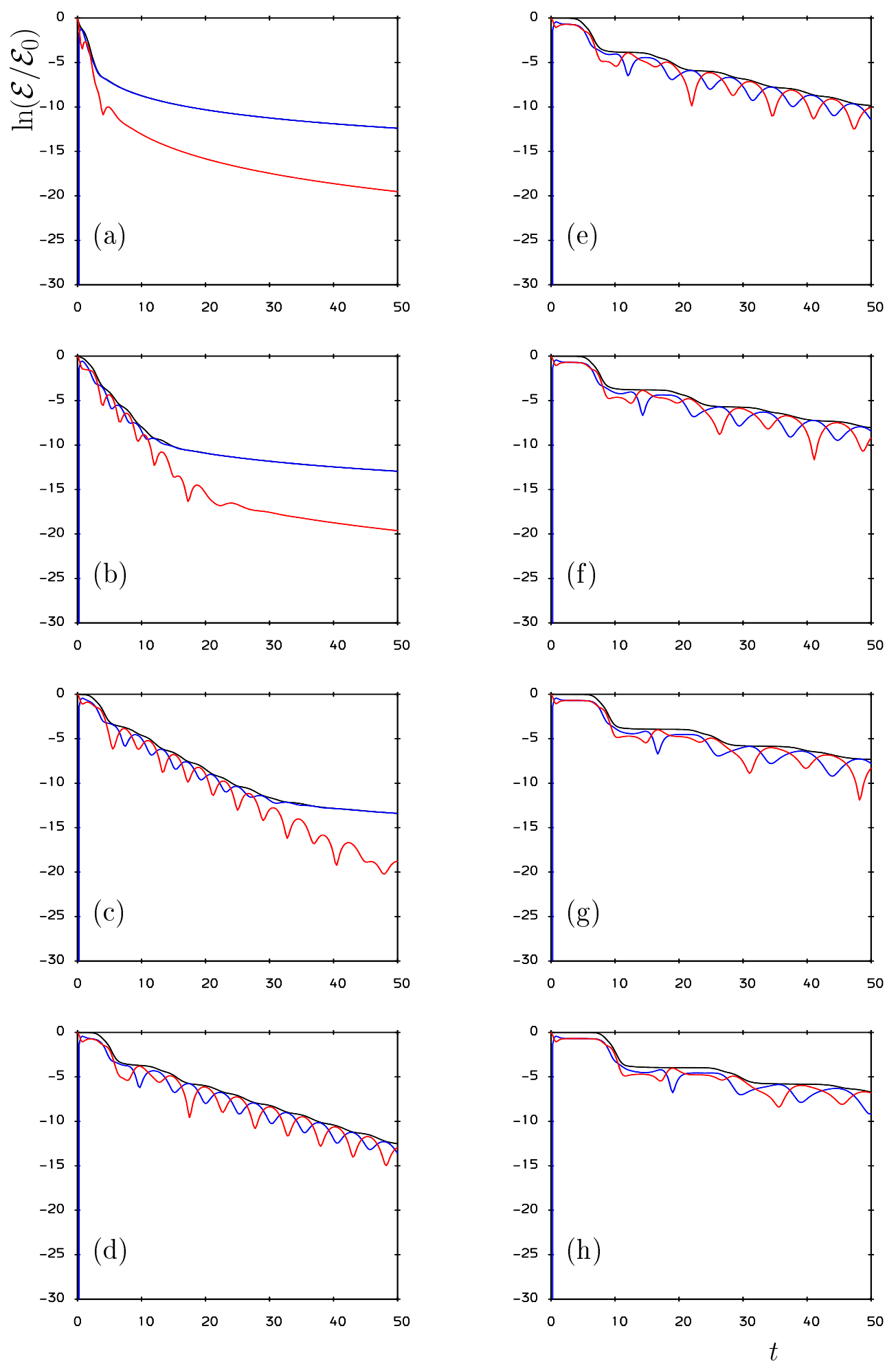

Figure 3: Time evolution of normalized total energy $\mathcal{E} / \mathcal{E}_{0}$ (black curves), field energy (red) and kinetic energy (blue) for $\delta_{e}=0$ and $S=10(\mathrm{a}), 10^{2}(\mathrm{~b}), 10^{3}(\mathrm{c}), 10^{4}(\mathrm{~d}), 10^{5}$ (e), $10^{6}(\mathrm{f}), 10^{7}(\mathrm{~g}), 10^{8}(\mathrm{~h})$. The initial conditions are $v=0$ and $\partial \psi / \partial r \propto \sin (\pi r)$ 

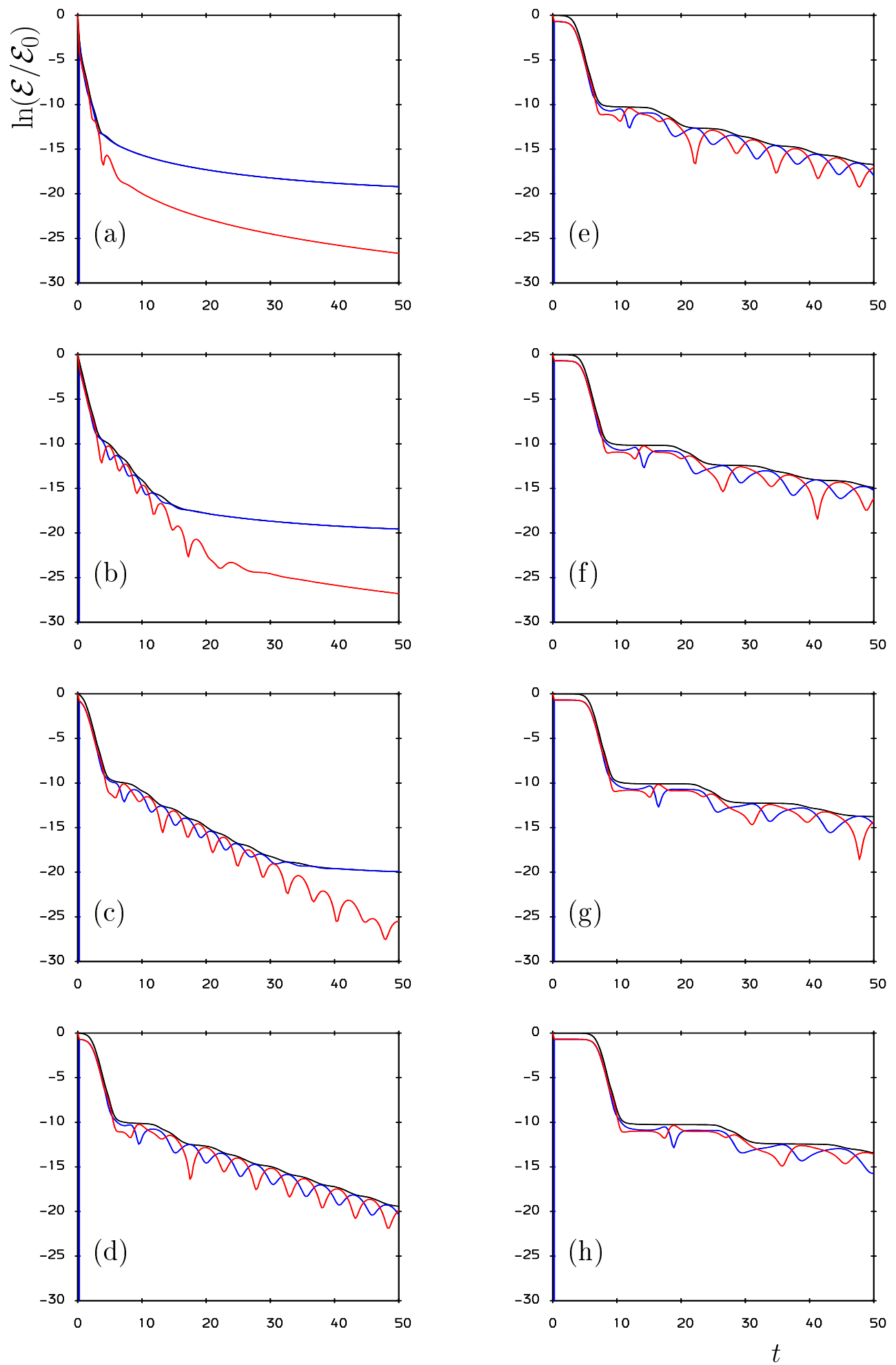

Figure 4: Time evolution of normalized total energy $\mathcal{E} / \mathcal{E}_{0}$ (black curves), field energy (red) and kinetic energy (blue) for $\delta_{e}=0$ and $S=10$ (a), $10^{2}$ (b), $10^{3}$ (c), $10^{4}(\mathrm{~d}), 10^{5}(\mathrm{e}), 10^{6}(\mathrm{f}), 10^{7}(\mathrm{~g}), 10^{8}(\mathrm{~h})$. The initial conditions are $v=0$ and $\psi \propto-1 / \exp \left[100(\ln r)^{2}\right]$ 

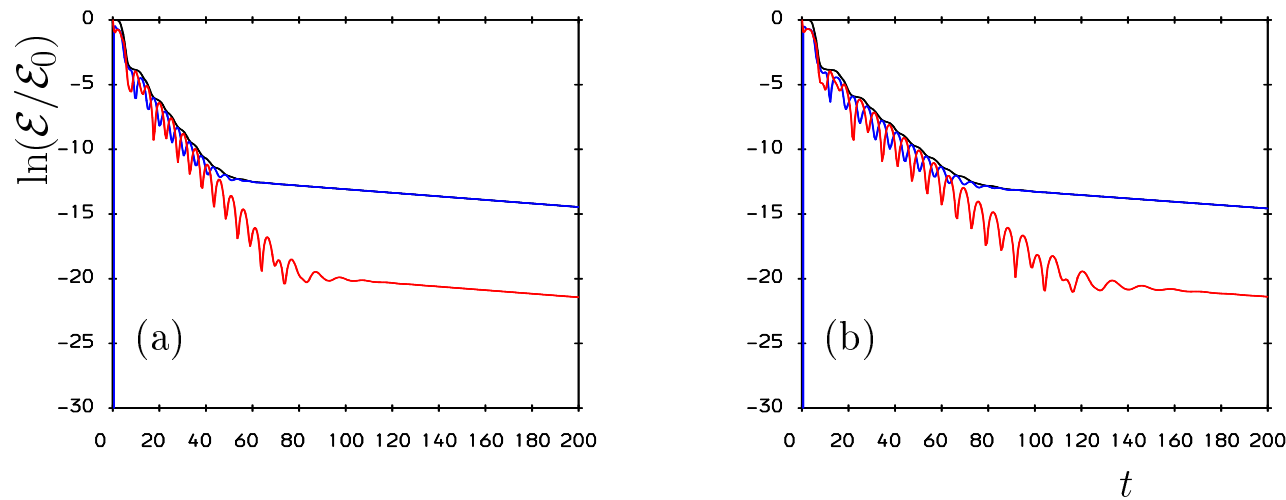

Figure 5: Time evolution over 200 Alfvén periods of normalized total energy $\mathcal{E} / \mathcal{E}_{0}$ (black curves), field energy (red) and kinetic energy (blue) for $\delta_{e}=0$ and $S=10^{4}$ (a), $10^{5}$ (b). The initial conditions are $v=0$ and $\partial \psi / \partial r \propto \sin (\pi r)$ 

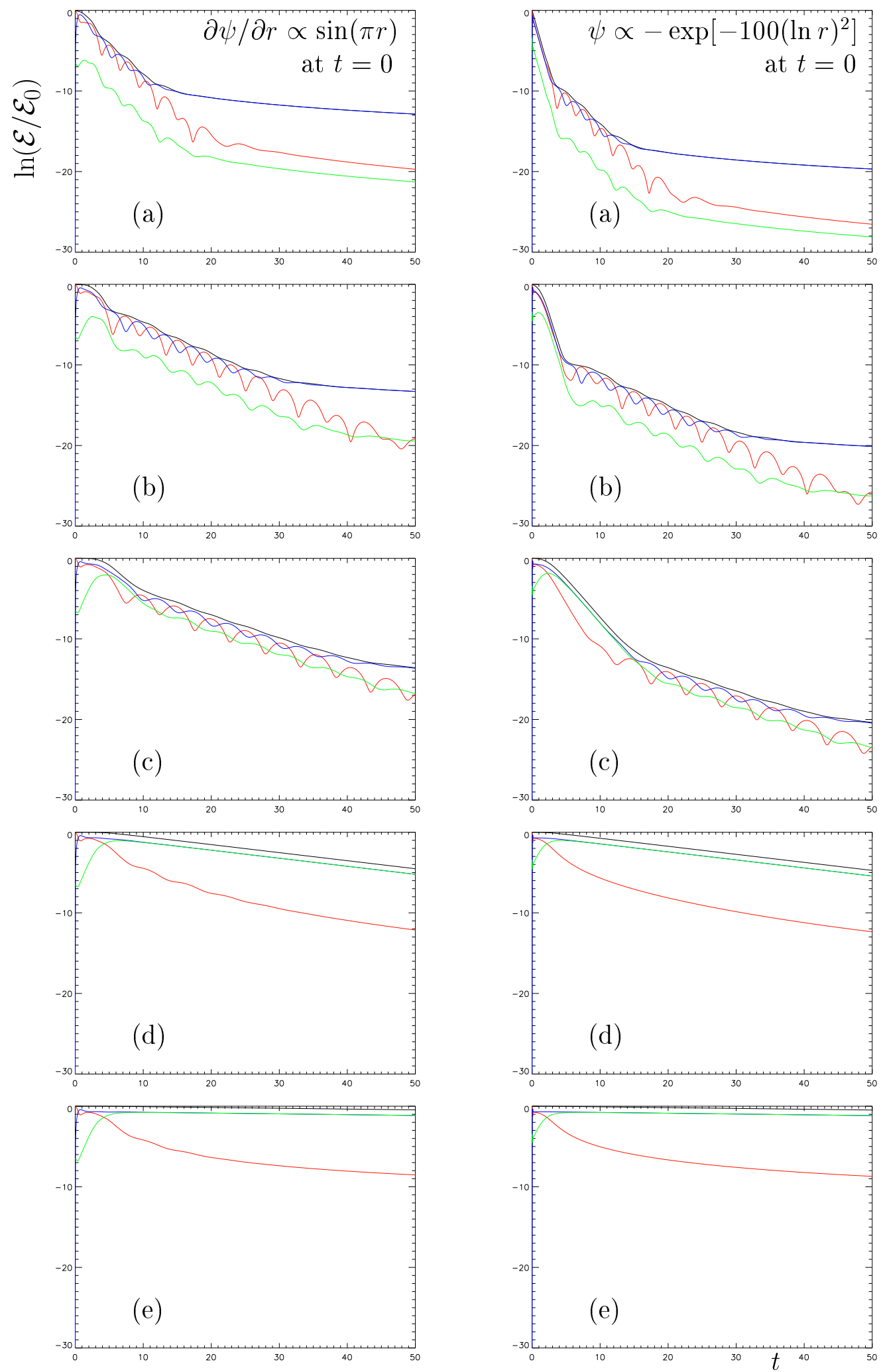

Figure 6: Time evolution of normalized total energy $\mathcal{E} / \mathcal{E}_{0}$ (black curves), field energy (red), ion kinetic energy (blue) and electron kinetic energy (green) for $\delta_{e}=0.01$ and (a) $S=10^{2}$, (b) $10^{3}$, (c) $10^{4}$, (d) $10^{5}$, (e) $10^{6}$. The initial conditions are $v=0$ and $\partial \psi / \partial r \propto \sin (\pi r)$ (left plots), $\psi \propto-\exp \left[-100(\ln r)^{2}\right]$ (right plots). 

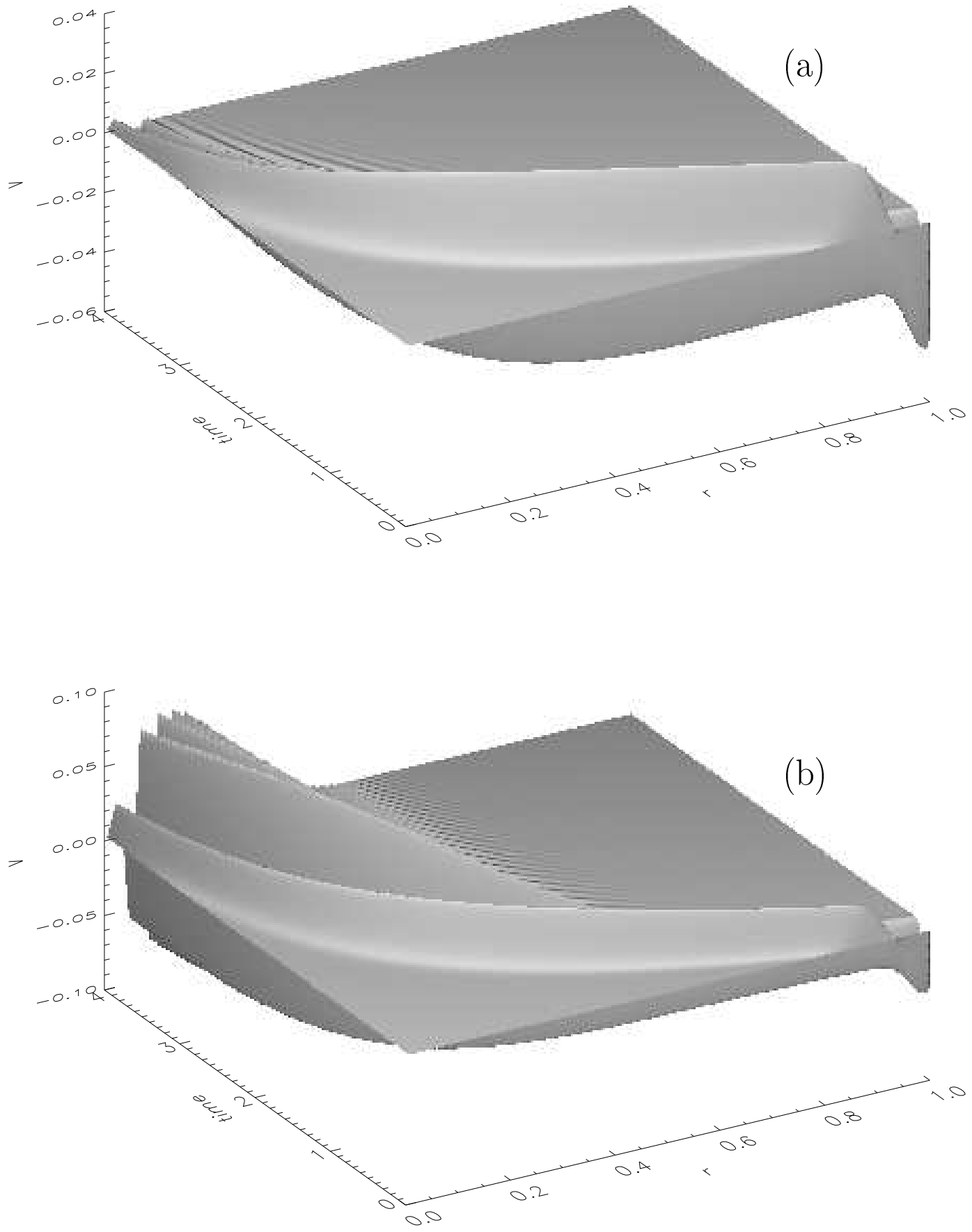

Figure 7: Space and time evolution up to $t=4$ of velocity in simulations with initial conditions $v=0, \psi \propto-\exp \left[-100(\ln r)^{2}\right], \delta_{e}=0.01$ and (a) $S=10^{3}$, (b) $S=10^{5}$. 

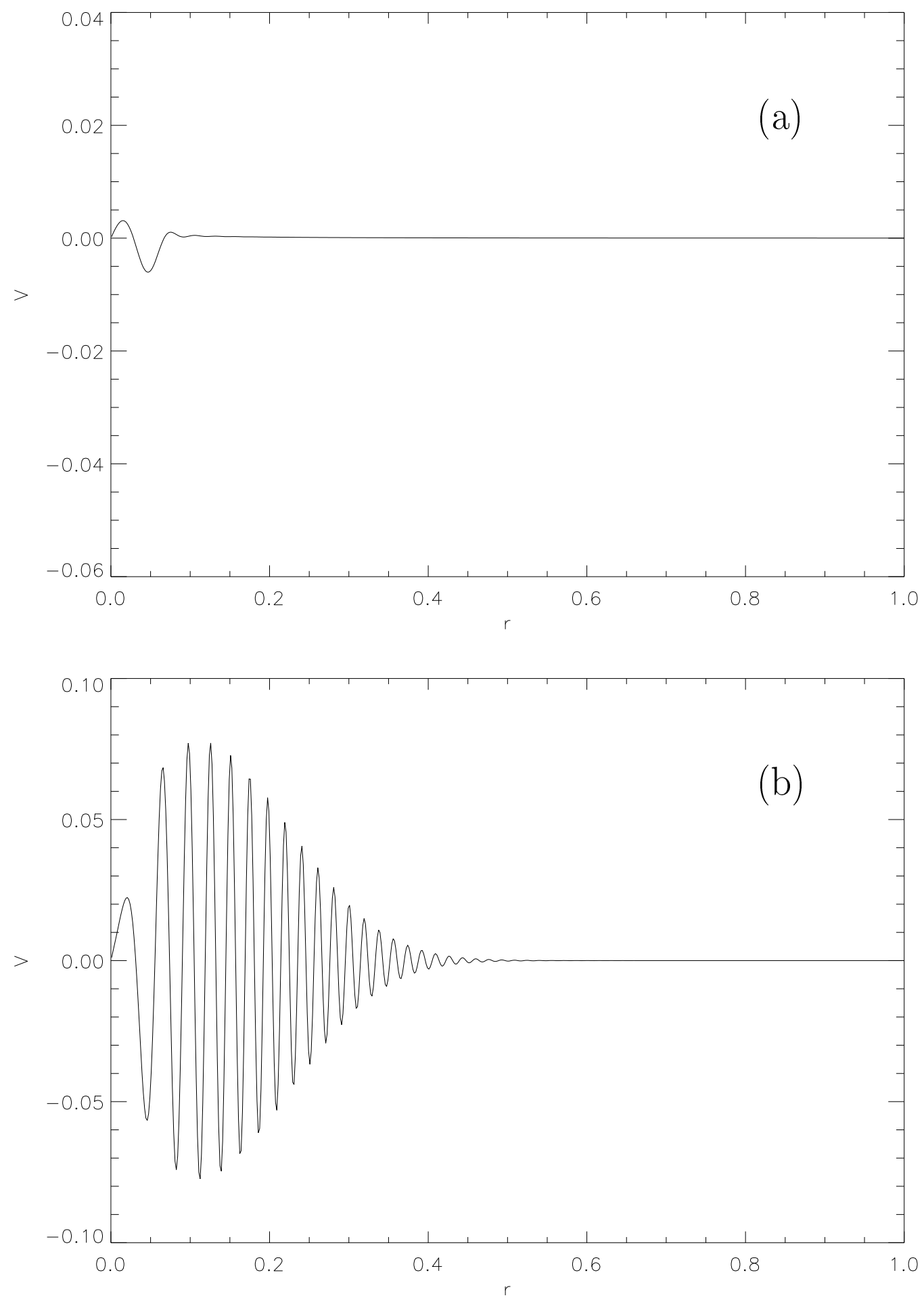

Figure 8: Velocity profile at $t=4$ in simulations with $\delta_{e}=0.01$ and (a) $S=10^{3}$, (b) $S=10^{5}$. The initial conditions are $v=0$ and $\psi \propto-\exp \left[-100(\ln r)^{2}\right]$. 